\title{
Research on a kind of energy interconnection communication network architecture based on the technology of SDN
}

\author{
Fengjie SUN ${ }^{1, a}$, Yanhong XU ${ }^{1, b}$, Geng ZHANG ${ }^{2, c}$, Xi LIU ${ }^{3, d}$ \\ ${ }^{1}$ Institute of Electrical and Electronic Engineering, North China Electric Power University, Beijing, \\ 102206, China \\ ${ }^{2}$ Institute of Information and Communication, China Electric Power Research Institute, Beijing, \\ 100192, China \\ ${ }^{3}$ Operation and Maintenance Center of Information and Communication, Sichuan Information and \\ Communication Company of State Grid, chengdu, 610041, China \\ aemail: sfj@ncepu.edu.cn, ${ }^{b}$ email: pearl1123581321@126.com, \\ cemail: zhanggeng@epri.sgcc.com.cn, demail: xliu_helen@hotmail.com
}

Keywords: Energy Interconnection; Communication Network Architecture; SDN Technology; Intelligent Energy Controller

\begin{abstract}
In this paper, we make use of the software defined network (SDN) technology's characteristic of separating the function of forwarding and control to concentrate control function in the control layer (comprised by SDN controllers), and design a kind of communication network architecture for energy interconnection. Firstly, we give the concept of Intelligent Energy Controller (IEC), and make it as the device to arrange information from underlying network and implement commands from upper layer. Then, we describe the architecture and running process of the system, by introducing in detail the design of the architecture and the flow of information flowing in the network and how to process them. At the end, we analyze the benefits and hidden dangers of the architecture, to reveal the advantages and disadvantages of the architecture, as a reference to related researches.
\end{abstract}

\section{Foreword}

The third industrial revolution written by the famous American economist Jeremy Rifkin causes governments' and scholars' concerning about the energy Internet. Scholars at home and abroad have done dozens of researches for energy Internet and related technologies, including the United States and Germany as the typical representative. FREEDM from American NSF and The Berkeley view are based on distribution characteristics of the American energy, and they are both a top-down and centralized management mode. Germany has also launched the E-Energy plan, and made the distributed and collaborative management model in Energy interconnection network according to the characteristics of regional integration in the European Union.

Some research institutions in China, such as Tsinghua University, BUPT (Beijing University of Posts and Telecommunications) and some other colleges and universities are also actively launched on the research of the energy Internet, and achieved considerable results. In this paper, on the basis of research results at home and abroad, we put forward a communication network architecture for energy interconnecting based on SDN technology. We will introduce the architecture in more details below.

\section{Intelligent Energy Controller}

First we give the concept of a new device called Intelligent Energy Controller (IEC). IEC mainly refers to the concept of IEM from FREEDM system, IPS from Berkeley view and the Energy manager from E-Energy.

The main functions of the IEC are to sort out the local information and sent them to the data 
center through the electric power communication network, and then control the local energy according to the return information from the data center.

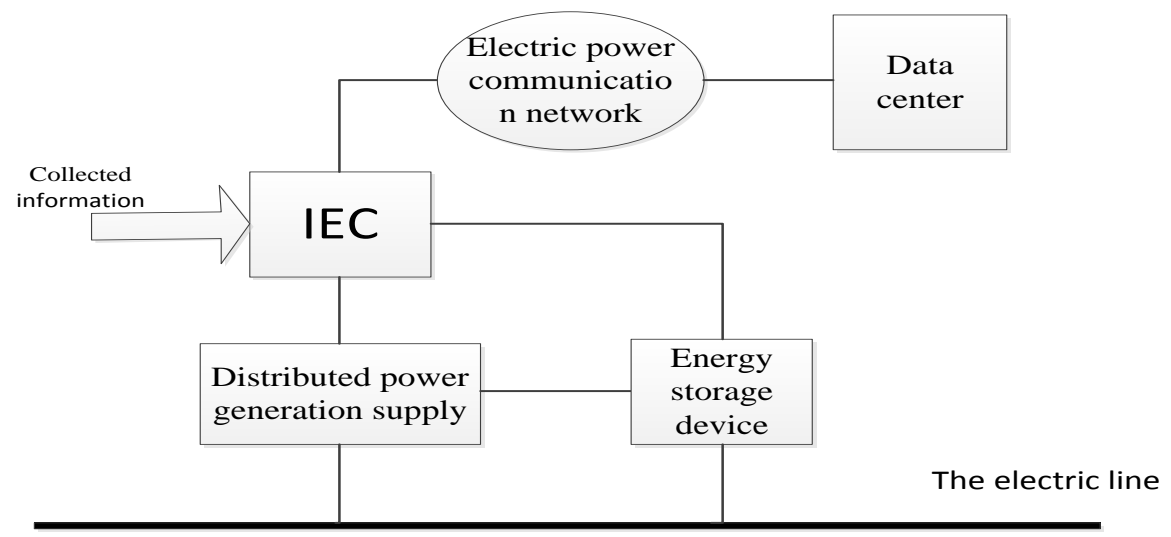

Fig.1. The position of IEC in the network

As shown in Figure 1, IEC will send the information (including those from the external acquisition device and internal information) to the data center after preliminary sorting. The data center will deal with the information, and then returns results to IEC. After receiving the results, IEC will control local distributed power generation supply and energy storage device according to these results. The distributed power generation supply showed in Figure 1 can be either larger renewable energy power generation supply, such as wind power plants, hydropower plants, etc., or small distributed power generation devices, such as some small energy collecting devices on buildings or in families.

These results from data center can be displayed on the IEC, and so local users can at any time to check the related information, including the amount of local already bought electricity and already sold electricity, cost to buy electricity and income from selling electricity, and whether local energy is invoked by others, and so on. IEC provides two selectable operation modes: manual mode and automatic mode. In automatic mode, the IEC will regulate local energy directly according to the results obtained from the data center. If local users feel the results from the data center would be wrong, such as user think in the next period of time the production of the local distributed power generation will be reduced, or power consumption peaks will come, and electricity may rise, so they don't want to let the power grid continues to call the local energy, or local energy users find abnormal call situation, such as no in but only leave, and which has affected normal local use of electricity, then the user can switch IEC to manual mode to order the IEC according to user's need, such as stop power supply outward or purchase power inward. In manual mode, the information from user's operations will be sent to the data center, and the data center will return corresponding processing results to response user's operations.

Above functions of IEC described can even be integrated into the ordinary PC. Basic components in an IEC include: a specially designed operating system, a display, some control buttons and necessary chips, such as information processing chip, memory chip, etc. The control of the physical parts (such as to electric power) can consult similar parts of functions of the energy router in FREEDM system, and we will not discuss it here.

\section{System Running and Network Architecture}

We mainly introduce the concept of IEC in front, and here we will describe in detail the overall framework of the whole energy Internet network and the running of the system.

Figure 2 is a diagram of regional energy interconnection network communication architecture, and SDN controller is used to control data centers.

Here is a brief introduction of SDN technology. As shown in Figure 3, SDN is divided into three layers: Bottom is the infrastructure layer made up of varieties of network equipment, such as routers and switches, and their control functions are abstracted to the control layer; The middle layer is control layer, its data plane interfaces to the south control network traffic in infrastructure layer 
through the special agreement, such as OpenFlow, while to the north it connects with various applications in the upper layer through the API; The top layer is the application layer, and in this layer, programmers can easily develop various applications which are necessary or wanted. SDN is a new type of network architecture put forward by the research team of clean slate of Stanford University. The core technology, OpenFlow, separates control plane and data plane, thus makes the flexible control of network traffic possible, and provides a good platform to innovating the core network and applications.

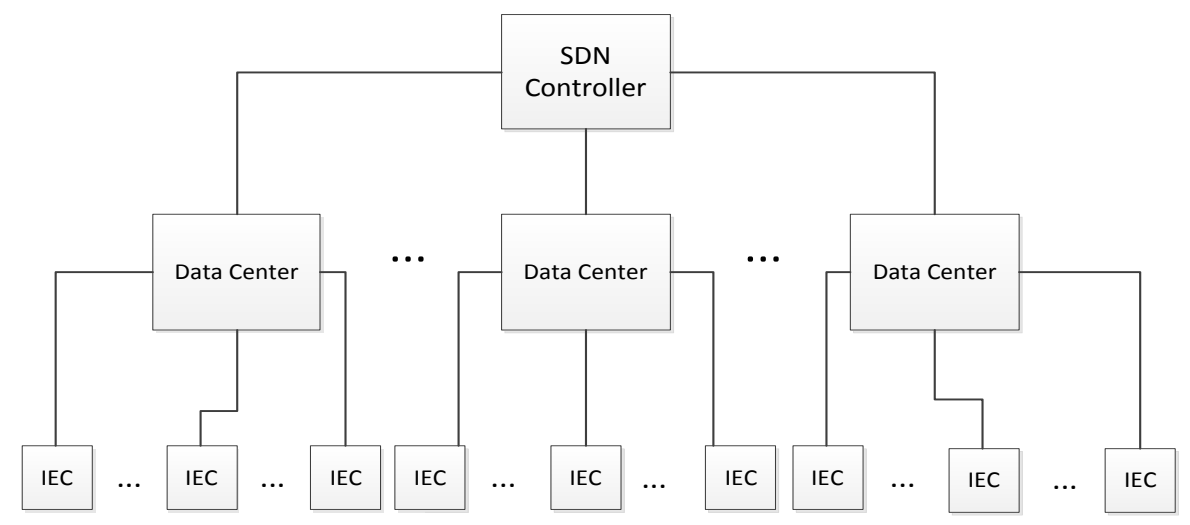

Fig.2. Diagram of Energy interconnection network communication architecture

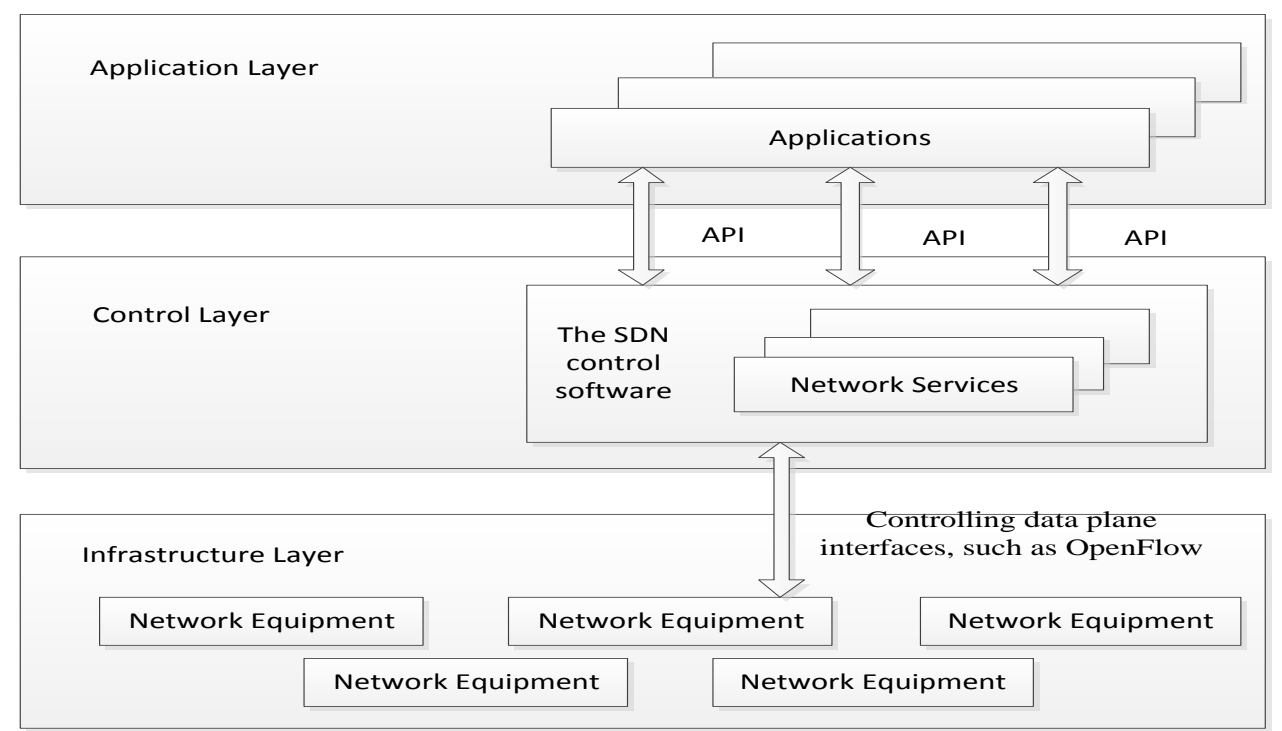

API: Application Program Interface

Fig.3. SDN architecture

Using SDN technology mainly because it's easy to change and manage applications, such as some algorithms (for example, load balancing algorithm) and information processing strategies, and it will be very convenient to improve these strategies in future.

The system is running as follows:

First of all, the acquisition devices transmit collected information to IEC. Here, acquisition devices can connect to the IEC by cable, or by wireless. And IEC will sort out the different types of such information to eliminate the differences of the underlying devices. The information includes local geographical location, local climate conditions (including current weather conditions and future weather conditions forecasted), local power generation (including the current power and average power over a period of time), local already sold electricity and already bought electricity, local energy storage, etc. IEC needs to put these information into a defined form, and then send the form to data center through the electric power communication network. The form here is a little similar to flow table of OpenFlow in SDN switches. The design of the form can make it convenient for data center to process the information fast. To correspond, the data center will define a return 
message form, and this form should include processing results of relevant information and some instructions, etc., to control the IEC. Schematic diagrams of the two forms are shown in Figure 4 and Figure 5.

\begin{tabular}{|c|}
\hline $\begin{array}{c}\text { Local geographical } \\
\text { location }\end{array}$ \\
\hline $\begin{array}{c}\text { Local climate conditions } \\
\text { Power generation of } \\
\text { local generation }\end{array}$ \\
\hline $\begin{array}{c}\text { Local already sold } \\
\text { electricity }\end{array}$ \\
\hline $\begin{array}{c}\text { Local already bought } \\
\text { electricity }\end{array}$ \\
\hline $\begin{array}{c}\text { Local energy storage } \\
\text { - } \cdots\end{array}$ \\
\hline Reservation extension \\
\hline
\end{tabular}

Fig.4. The form uploaded from IEC

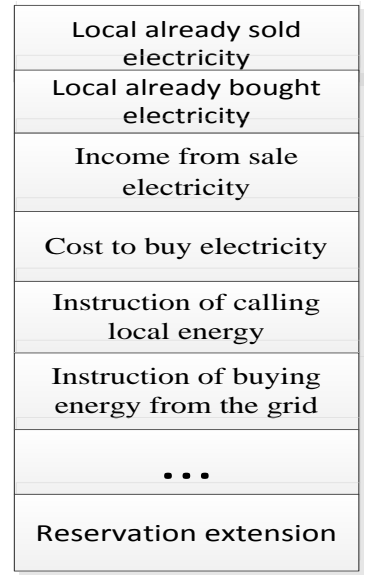

Fig.5. The form returns from Data center

Later, the data center will process received information. Data center will set up one virtual machine for each IEC to store and manage their information conveniently, and the SDN controller will build a geographical distribution map of IECs according to the information of locations stored in the virtual machine, to use nearby principle to call energy. In addition, the controller will calculate uploaded data of every IEC to make analyses, including the productivity prediction according to information of local climate conditions, predicting the future electricity utilization according to information of local already used electricity, providing electricity bills according to the information of buying and selling energy, giving orders to buy or sell according to local production capacity and energy storage and the usage of electricity in other areas, etc., and then the results will be sent back to the corresponding IEC. The controller and data centers linked can communicate with each other by OpenFlow protocol.

After receiving results from data center, IEC will perform some operations, such as whether to call local energy. We've mentioned that the IEC has two modes, and normally it will enable automatic mode, when the IEC will implement the operations directly in accordance with the returned information.

In addition, SDN controllers in different regions can connect to achieve a wider range of energy regulation.

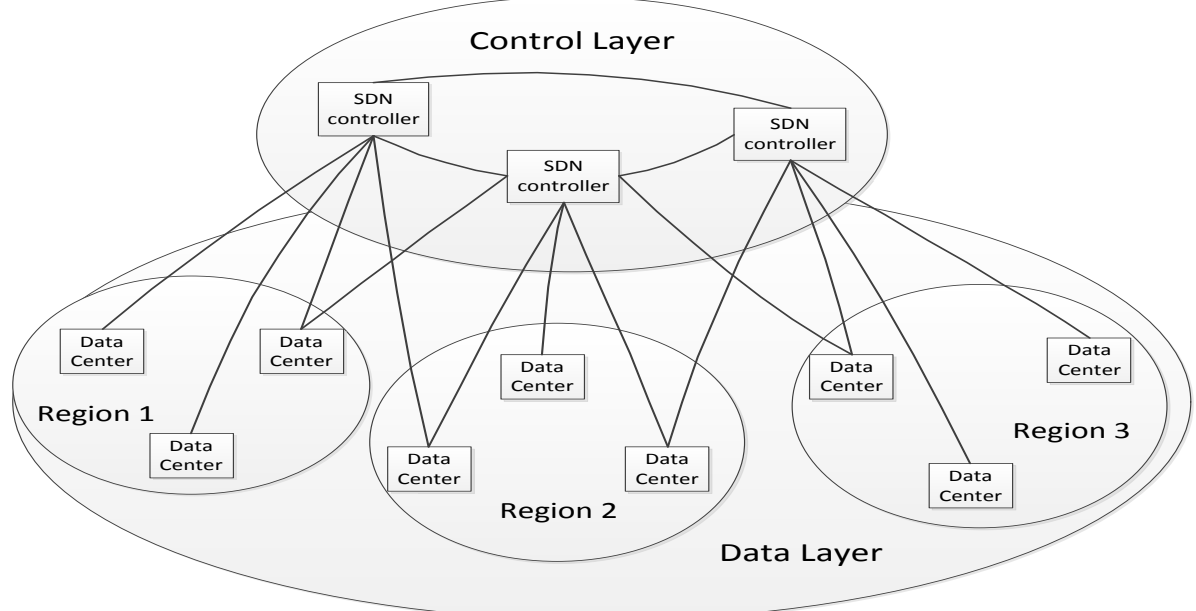

Fig.6. Connection diagram of SDN controllers and data centers

Figure 6 shows connection diagram of SDN controllers and data centers. In order to enhance the reliability of the network, a single SDN controller not only connects with data centers in its' own jurisdiction, but also some other data centers in neighboring regions to be their backup controller. By this kind of connection, each data center links at least two SDN controllers. But one data center 
will only communicate with one controller at the same time, if not so, one data center taking up more than one controller at the same time will cause the waste of resources and more than one controller sending different instructions to the same data center at the same time will lead to decision conflict. So it will need some load balancing algorithms here to calculate and determine each controller will control which data centers at the certain time.

The standards of east-west interface used for multiple SDN controllers to communicate have not been defined, and here we can use cluster technology to solve this problem. The reference [5] points out that the server can be used as a carrier of the controller as a controller just likes a software system, thus the controller cluster can be set up based on server cluster.

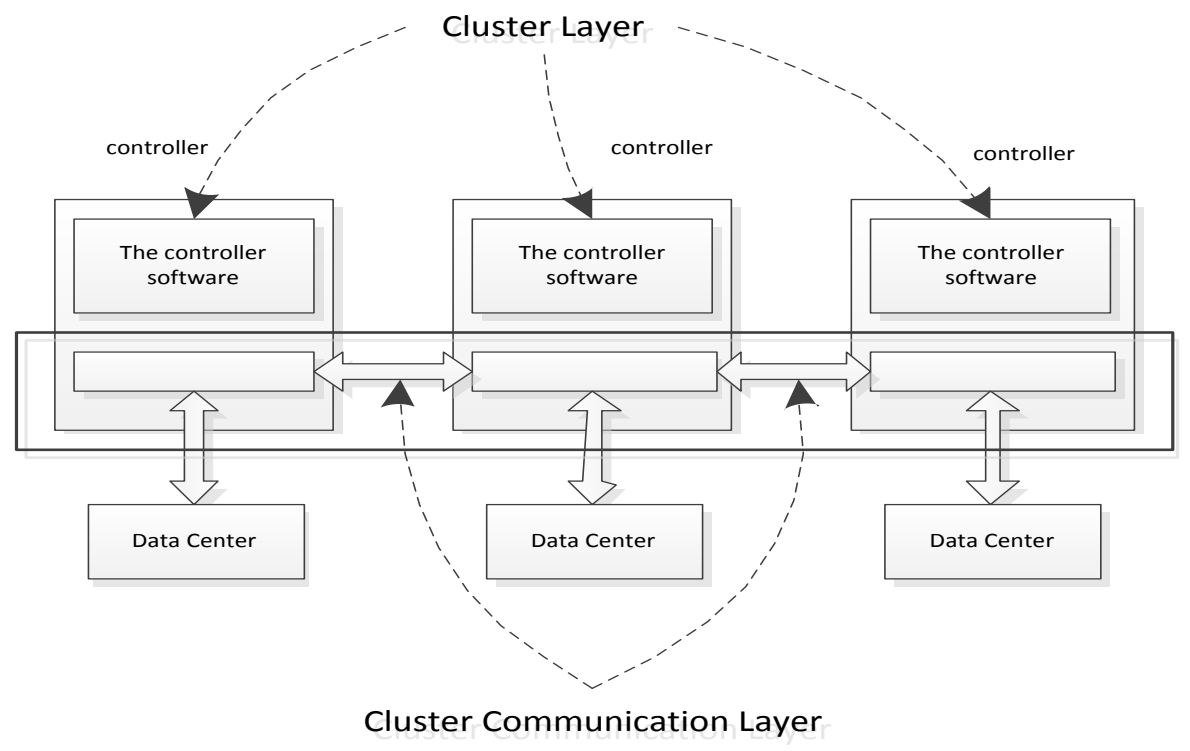

Fig.7. Controller cluster architecture

Figure 7 is the diagram of controller cluster architecture designed based on the literature [5]. According to the literature, SDN can make full use of the server cluster technology (such as Hazelcast) to deploy the controllers realized by software. And for a distributed cluster system, the collaboration between controllers matters very much, so the communication mechanism in the cluster system needs to be specially set up, and the existing communication library such as JGroups is a very mature technology.

At present, the controller cluster technology is a very good choice to solve the problem of the coordination of multiple controllers. And we believe that when the east-west interface standards of SDN controllers defined, the collaborative between controllers and openness and scalability of the control layer will all be able to be improved.

\section{Characteristics Analysis}

The biggest characteristic of this architecture is to hand over tasks of scheduling energy directly to the SDN controllers, and this will bring two advantages:

1) The SDN controller in the top layer has the most comprehensive knowledge about energy distribution and the demand of the whole area, so such a macro regulation and control should be able to make the most reasonable energy scheduling policy;

2) IECs in the bottom layer do not need to communicate with each other, thus there's also not need to define the communication protocols between them. The IECs' job is to control local energy according to the orders from the controllers or users. So the requirements of communication and information processing to the bottom devices will be reduced.

Meanwhile, the system will have some hidden worries as well:

(1) The responsibility of SDN controller will become very grave for all energy scheduling will be arranged by it, as a result, the processing capacity and efficiency of SDN controller becomes a 
very important indicator. At the same time, the rationality and validity of algorithms and strategies in the controller will directly impact on the rationality of power dispatching over the whole area. And problems will ensue, such as how often IECs should upload information to the controller, how to design the two forms in detail mentioned above, and so on. All these problems need further researches.

(2) Setting two modes in the IEC is originally in order to guarantee the stability of the local electricity and increase the user's choice. But if distributed energy sources become main sources of energy in the power grid in the future, and there're too many users take unreasonable control on local energy, that may cause the difficulty for the controllers to call the energy, and leads to the unreasonable scheduling of energy. So there is also need to consider such options a second time.

\section{Summary}

Energy is a basic support of human production activities, and lots of attention has been paid to environmental problems caused by the traditional energy. Now its own shortage of energy is also becoming increasingly obvious, and human has to attach importance to the fact of it. Clean and infinite renewable energy, such as wind, hydro, solar, biomass energy and ocean energy, etc., although researched for a long time, still can't become main sources of energy. Under this embarrassing situation, Jeremy Rifkin put forward to interconnect different types of energy using information and communication technologies, to allocate existing energy reasonably, and make renewable energy gradually replaces the traditional energy, so as to realize the effective utilization of energy and the production of sustainable. The concept of energy interconnection provides a direction for the development of energy in future, and points out an advisable way for energy problems. It also gives us a hope of a new different future.

We all wish to contribute to the research of energy Internet. This paper just gives one conception of the architecture of energy internet, and hope to provide a reference for the research of the energy Internet.

\section{References}

[1] A Huang, M L. Crow, G T Heydt, et al. The Future Renewable Electric Energy Delivery and Management (FREEDM) System: The Energy Internet[C]. Proceeding of the IEEE, 2011, 99 (1):133-148.

[2] R H Katz, D E Culler, S Sanders, et al. An information-centric energy infrastructure: The Berkeley view [J]. Sustainable Computing: Informatics and Systems, 2011, 7:7-22.

[3] Xie Tao. Research on architecture and key technologies of energy internet [R]. Postdoctoral research reports of Tsinghua University, 2013.

[4] Ludwig Karg. E-energy projects and ancillary research [R/OL]. 2014-7-21.

[5] An exploration to the SDN architecture based on controller cluster [J/OL]. http://www.d1net.com/virtual/tech/247954.html, 2013-12-19.

[6] Jeremy Rifkin. The third industrial revolution [M]. CITIC Press, 2012, the first edition. 